\title{
An Approach for a National eHealth Implementation - the Case of Modular Interactive Tiles for Rehabilitation
}

Lund, Henrik Hautop ; Jensen, Line Steiness Dejnbjerg; Ssessanga, Yusuf; Cataldo, Stefano; YahyaMalima, Khadija I.

Published in:

IST-Africa 2015 Conference Proceedings

Link to article, DOI:

10.1109/istafrica.2015.7190552

Publication date:

2015

Document Version

Publisher's PDF, also known as Version of record

Link back to DTU Orbit

Citation (APA):

Lund, H. H., Jensen, L. S. D., Ssessanga, Y., Cataldo, S., \& Yahya-Malima, K. I. (2015). An Approach for a National eHealth Implementation - the Case of Modular Interactive Tiles for Rehabilitation. In P. Cunningham, \& M. Cunningham (Eds.), IST-Africa 2015 Conference Proceedings https://doi.org/10.1109/istafrica.2015.7190552

\section{General rights}

Copyright and moral rights for the publications made accessible in the public portal are retained by the authors and/or other copyright owners and it is a condition of accessing publications that users recognise and abide by the legal requirements associated with these rights.

- Users may download and print one copy of any publication from the public portal for the purpose of private study or research.

- You may not further distribute the material or use it for any profit-making activity or commercial gain

- You may freely distribute the URL identifying the publication in the public portal 


\title{
An Approach for a National eHealth Implementation - the Case of Modular Interactive Tiles for Rehabilitation
}

\author{
Henrik Hautop LUND ${ }^{1}$, Line S. D. JENSEN ${ }^{1}$, Yusuf SSESSANGA ${ }^{2}$, \\ Stefano CATALDO ${ }^{3}$, Khadija I. YAHYA-MALIMA ${ }^{4}$ \\ ${ }^{1}$ Technical University of Denmark, Building 326, 2800 Kgs. Lyngby, Denmark \\ Tel: +45 45253929, Email: hhl@playware.dtu.dk \\ ${ }^{2}$ University of Iringa, P.O.Box, 200,Iringa, Tanzania \\ Tel: +255(026)2720900, Fax: + 255(026)2720904,Email:iuco@tumaini.ac.tz \\ ${ }^{3}$ INUKA Inuka Southern Highlands Community Based Rehabilitation Program, \\ Waging'ombe, Njombe, Tanzania, Email: stefanocataldo@yahoo.it \\ ${ }^{4}$ Tanzania Commission For Science and Technology (COSTECH), P.O. Box 4302, \\ Ali Hassan Mwinyi Road, Kijitonyama (Sayansi) COSTECH Building, \\ Dar es Salaam, Tanzania
}

Tel: Office +255 22 2927538, Email: kmalima@costech.or.tz

\begin{abstract}
By the development of a mHealth tablet app together with modular interactive tiles for rehabilitation, we intend to facilitate the co-design, adaptation, demonstration and validation of modular ICT solutions for rehabilitation in deep rural sub-Saharan Africa. This results in highly mobile, modular and energy efficient technology which can be set up and used anywhere and anytime. We have formed a national partnership for sustainable implementation, comprising a governmental representative, national hospital, national health university department, regional hospitals, Living Labs and NGOs performing community-based rehabilitation. Thereby, we investigate the adaptation, contextualisation and implementation in different rehabilitation methods and centres, including hospitals both in a city centres and in a rural area, NGO's performing community based rehabilitation, and rehabilitation centres. Together, the partners contextualise the eHealth solution to fit the needs in urban, rural and deep rural areas.
\end{abstract}

Keywords: eHealth, rehabilitation, playware, modular tiles.

\section{Introduction}

mHealth is often focused on health promotion, health education, disease prevention, and monitoring, and little evidence is found on using mHealth directly as a tool for health intervention and rehabilitation. For instance, the recent systematic review on mHealth in Africa found no studies addressing rehabilitation [1]. As an eHealth tool extended to become a mHealth system for rehabilitation, we created the modular interactive tiles as electronic building blocks that can be brought to anybody, anywhere, anytime. Just like LEGO, the modular interactive tiles can be put together in any configuration to suit our needs and interests, for instance in rehabilitation of stroke patients and children with cerebral palsy. Indeed, the modular interactive tiles are developed as an alternative form of physical rehabilitation exercise. With this new technology, users are able to break away from monotonous treatment programmes and participate in exercise that is fun and exciting, and therefore more motivating. The battery-powered tiles present a vast number of opportunities for games and can be used for many different purposes. Indeed, due to its 
modularity - like LEGO - it is very easy to co-design and adapt the modular tiles system to different contexts, different intervention areas, and different levels of competencies.

This can have an important impact in rehabilitation of disabled, stroke, and cardiac patients in sub-Saharan Africa where a large part of the population lives in remote and rural areas. Here, potential health technology solutions often face problems of scarce availability of electricity, of technology professionals, and of health personnel. Further, due to the relatively low amount of health spending and of health workers in sub-Saharan Africa compared to high-income countries, the individual health worker will typically need to treat a vast array of health problems compared to the high degree of specialization to single health problems amongst health workers in high-income countries. Hence, to be effective in sub-Saharan Africa, there is a need for health technology solutions which facilitate treatment of people with various diagnoses.

We try to addresses this challenge with the modular interactive tiles, which aim at motivating patients to perform rehabilitative actions. This motivational approach has shown its validity and high potential in Europe, and initial pilot studies in sub-Saharan Africa show similar promise (as reported in IST Africa 2012 [2] and IST Africa 2014 [3]). By taking advantage of the flexibility and easy approach to personalization inherent in the modularity, the proposed modular approach possesses the potential of becoming particularly helpful in addressing both the technological challenges and health challenges in sub-Saharan African communities. With the high prevalence of disability, stroke and coronary heart cases in sub-Saharan Africa countries, it is of paramount importance to investigate how this kind of an approach can provide an eHealth solution flexible enough to adapt to the needs of patients. We therefore aim at refining and contextualising modular interactive tiles to allow a local community to have one modular solution that any community rehabilitation worker can easily adapt to the individual patient and his/her needs. This contextualisation and adaptation is further facilitated by the development and introduction of a tablet app for the modular interactive tiles, see Figure 1. The Android tablet app allows easy adaptation and co-design of games, levels, immediate feedback, and documentation. The immediate feedback can be in the form of score shown visually and sound as notes, spoken words, etc.



Figure 1. The tablet app is developed to easily control games on modular tiles and to allow different kinds of feedback (e.g. score and sounds as immediate feedback, and collection of score on the internet, e.g. to document health effect from using the modular tiles) . 


\section{Objectives and Methodology}

Through well-structured national partnerships, we aim ti implement this concept in SubSaharan Africa. In developed nations, games for health [4, 5] and exercise-games [6, 7] have shown potential for motivational interventions in many different health areas. For instance, previous studies have shown the potential of using modular interactive tiles for activating mentally handicapped children [8, 9], hospitalized children [10], kindergarten children, cardiac patients [11, 12], and stroke patients [13, 14]. Since the Sub-Saharan African environment has a very different infrastructure, a novel approach for implementation of such an eHealth solution needs to be developed. This happens by establishing a strategic partnership in each of the main participating countries. Each national partnership comprises a governmental representative, national hospital, national health university department, regional hospitals, Living Labs and NGOs performing community-based rehabilitation. Thereby, we investigate the adaptation, contextualisation and implementation in different rehabilitation methods and centres, including hospitals both in a city centres and in a rural area, NGO's performing community based rehabilitation, and rehabilitation centres. Together, the partners contextualise the health technology solution by refining the software, games, solar recharging mechanism, minimizing need for support, etc. to fit the needs in the implementation areas, and make larger qualitative and quantitative studies of the effect of implementing the modular tiles for rehabilitation in hospitals, rehabilitation centres and in community-based rehabilitation in urban, rural and deep areas. Further, for the wider implementation in Sub-Saharan Africa in the future, it is the aim to include a number of associated countries (5-6 countries in sub-Saharan Africa) in the region to perform investigation of the health care system, its needs and requirements, and the potential for implementing the modular ICT system validated through effect studies in the initial, main participating countries. We try to generalise this knowledge into a coherent strategy through the collaboration with the governmental representatives and health authorities for the implementation of the rehabilitation approach with this modular health technology.

Consequently, we aim at providing everyone, regardless of their location, with better and personalised healthcare. At the same time, we cut down the cost for health care systems by contextualising and exploiting a solution that can be used by anybody to help treating a vast variety of physical and mental health rehabilitation challenges in low income countries in sub-Saharan Africa. As a green technology, by the development of an energy efficient solution that allows the modular tiles to run with minimal energy consumption, we aim at consuming less than $1 \%$ of energy of a normal exercise treadmill, allowing quick recharging with small solar cells in rural and deep areas of Sub-Saharan Africa. Finally, we investigate the development of a simple documentation of effect solution which will require minimal bandwidth allowing public authorities in a faster and more effective way to document and evaluate the effect in the health interventions. The system will allow the user to manage and protect their identities and personal data by providing anonymous profiles and access control.

Hence, our methodology is based on including the partners relevant for making a sustainable implementation in each country, starting from ministries and governmental representatives, and including all layers of the health system from national hospitals in the urban areas to community-based rehabilitation in deep, rural areas, see Figure 2. 


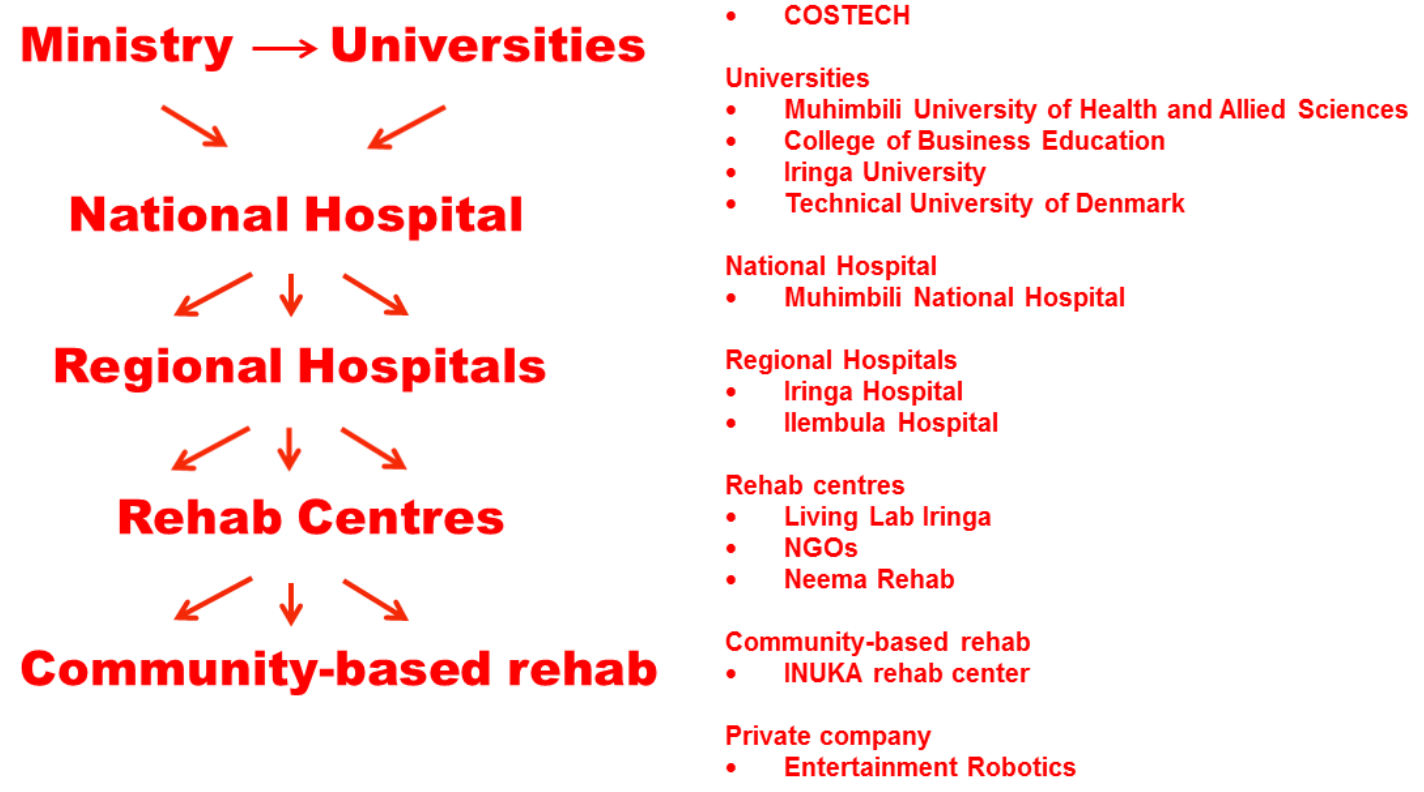

Figure 2. Left: Structure of Strategic National Networks. Right: Example of Partners in Tanzania.

\section{Location and Test Set-up}

\subsection{National Hospitals}

There are, in total, four National Hospitals in Tanzania, which are centralised in the largest cities in the country. These hospitals are the best, most advanced hospitals in the country, thus this is where sick patients get referred to by their Regional or District hospitals. These hospitals have the best educated staff with many specialists to take care of all kind of diseases and disabilities. The hospitals have rather good equipment and both power and back-up power. Their facilities are good and versatile, giving them the possibility for good treatment for their patients.
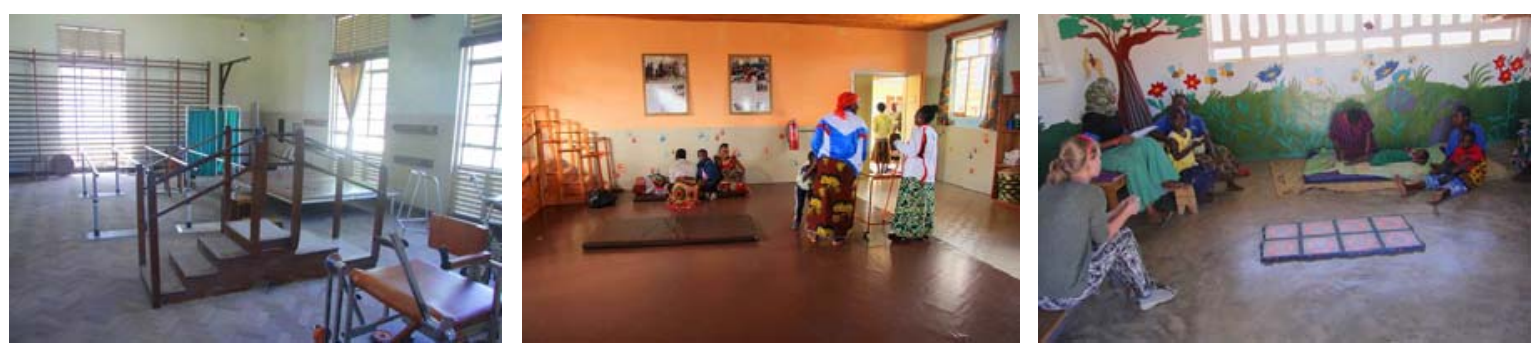

Figure 3: Left: Physiotherapy Department at Muhimbili National Hospital. Middle:

Rehabilitation Centre Inuka in Wanging'ombe. Right: Communal Rehabilitation Centre in Ilembula

\subsection{Regional and District Hospitals}

There are 18 regional hospitals and around 130 district hospitals. These are scattered in the regions and districts, thus bringing them out to the more rural areas as well. The employees are not as specialised as in the national hospitals, and many patients will thus be referred to the centralised national hospitals instead of the regional or district hospitals. The staff are not specialised, but there are some specialised staff at the regional hospitals, since they are bigger. The facilities are not as versatile, but include $\mathrm{x}$-rays equipment and more commonly used equipment. The staff have some education. 


\subsection{Rehabilitation centres}

The rehabilitation centres are often placed in small rural areas, where there is limited access to governmentally run hospitals. The staffs are often from western countries, locals with higher education, or locals who have been trained to do specific tasks. The Rehabilitation centres focus on physiotherapy and occupational therapy, for rehabilitating users with disabilities. The facilities are limited with only little specialised equipment, but have light, water and stable power.

\subsection{Communal Rehabilitation centres}

The communal rehabilitation centres are smaller and placed in more rural areas than the rehabilitation centres. The staff here has mostly only gotten some training from the rehabilitation centres in order to be able to execute the needed exercises with the patients. These centres are often small and without water and electricity.

\section{Technology Description - Modular Interactive Tiles}

To meet the demands in these urban and rural locations, we developed the system of modular interactive tiles. The tiles are designed to be flexible and in a motivating way to provide immediate feedback based on the users' physical gaming interaction, following design principles for modular playware [14].
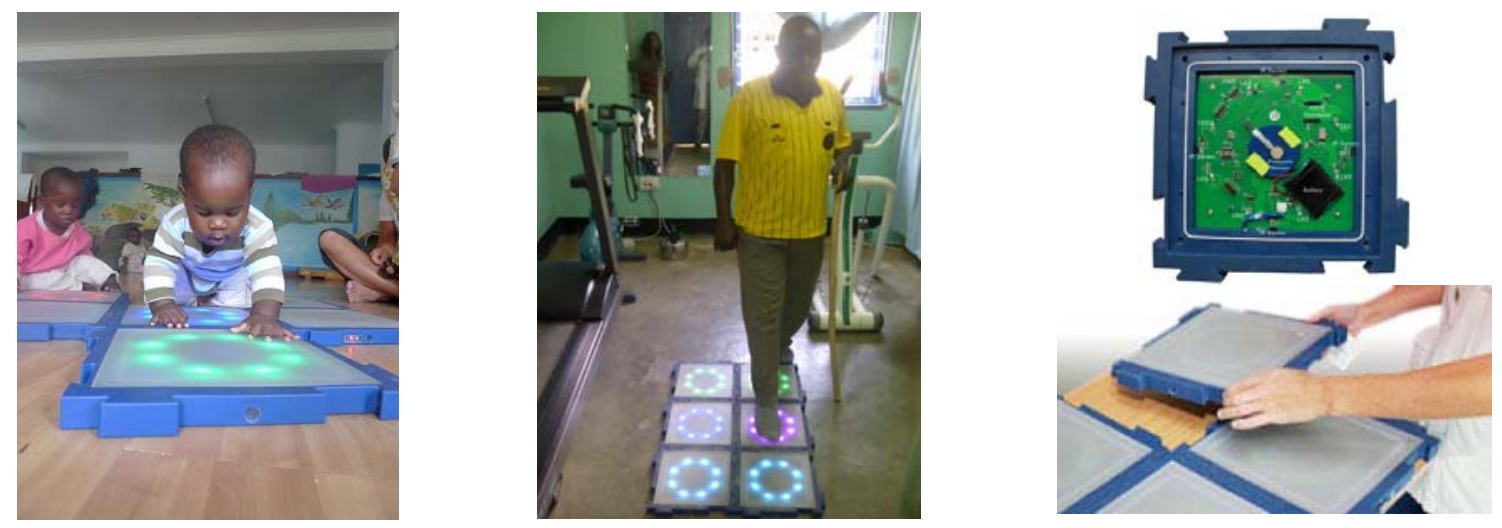

Figure 4: Modular Tiles Used for Hand or Feet Interaction

Each modular interactive tile has a quadratic shape measuring $300 \mathrm{~mm} * 300 \mathrm{~mm} * 33 \mathrm{~mm}$ - see Fig. 4. It is moulded in polyurethane. A system composed of modular interactive tiles is a fully distributed system, where each tile contains processing (ATmega 1280), own energy source (Li-Io polymer battery), sensors (FSR sensor and 2-axis accelerometer), effectors (8 colour LEDs), and communication (IR transceivers, and possibly XBee radio chip). In this respect, each tile is self-contained and can run autonomously. The overall behaviour of the system composed of such individual tiles is however a result of the assembly and coordination of all the tiles. The modular interactive tiles can easily be set up on the floor or wall within one minute. The modular interactive tiles can simply attach to each other as a jigsaw puzzle, and there are no wires. There are numerous games for both physical and cognitive training.

The modular interactive tiles are individually battery powered with a rechargeable Li-Io polymer battery, which can run continuously for approximately 30 hours and takes 3 hours to recharge - an important long battery life for the utilisation in rural areas in developing countries. The battery has to be charged once in a while, but can then often run for a week if the tiles are used e.g. 3-4 hours a day. 
We developed a tablet app, which allow the user to easily select games, level, and duration, as well as to monitor score and progress on the tablet (see Figure 1). At the same time, the tablet app is developed to provide feedback at run-time in the form of score presented on the tablet and sound emitted from the tablet. The tablet app runs on Android, and the tablet communicates with the ANT+ protocol [15] to the modular interactive tiles.

\section{Results}

Qualitative data was collected from a study with the aim of identifying the pros and cons using the modular interactive tiles for rehabilitation throughout an example national network of national hospital, regional hospital, rehabilitation centres and community rehab centre in Tanzania. This was done with different user groups to test for the versatility of the approach.

\subsection{Rehabilitation Centre and Community-based rehab: Inuka CBR}

At the rehabilitation centre Inuka CBR in Waging'ombe, the tiles are used with a variety of children. Through interviews and questionnaire with physiotherapist Raya and a group of volunteers, the following observations were made.

At Inuka CBR, the modular interactive tiles are used for physical play with the aim to facilitate the development of balance, coordination, memory and concentration. In hemiplegic cases, the patients are stimulated to use paretic limb, improve their foot support and to increase joints range of motion.

The modular interactive tiles are not used just for physical disabilities but also for mental disorders. Due to the bright colors of its lights and the quick lighting up of different areas, the patients with autism or ADHD are particularly attracted and consequently more stimulated to concentrate. As a result, Inuka CBR evaluates that this game has the capacity to increase the attention span, helping the therapist build their relationship with autistic patients.

Also in the tetraplegic cases with visual impairment, the use of tiles in a shady room, allows the user to identify easily their target, motivating the player to perform specific physical actions and goading the use of hands.

Inuka CBR evaluates that the modular interactive tiles is an intuitive and innovative game that can be used for physical disabilities both severe or not and also for mental and behavioral disorders. The possibility of using the game by varying the number of tiles, consents to customize it, depending on the patient impairment. As a result the game can be considered a good rehabilitative tool for playful physiotherapy providing to the individual user motivation to perform physical activities.

The activities with the tiles are implemented usually at Inuka Rehabilitation Center during a week of intensive treatment or during individual sessions. This allows the facilitators to use the tiles more times with the same child, which is very important in order to get a clearer picture of how the child progresses with the tiles. Testing with the children several times is also important, because many of the children who are mentally retarded need time to get acclimated to the new equipment. In the current form, it has been viewed to be more difficult to implement activities in the Community Rehabilitation Centers. The tiles are used mainly by therapists and still there are no Community Rehabilitation Workers able to use them independently. Also, in the current form, the facilities and infrastructure of Inuka Headquarters are more adequate to use the tiles. Figure 5 shows an example of observations by the physiotherapist Raya from different cases with some child patients using the modular tiles system, indicating some opportunities and challenges. 




Case 5: Autism; speech impairment

Game: flash paint; Colour race

Result: Went extremely well; child interested in the game; spoke a few words as she was playing; was able to interact with other child when playing (something that usually doesn't happen).

Problem: When playing colour race did not have enough strength to press the tiles.

Case 6: Burn

Game: Colour race

Result: Child enjoyed the game; helped him improve hand strength

Problem: Child didn't have enough strength to press the tiles

Case 7: Cerebral palsy and mental retardation

Game: colour race; concentration; paint

Result: Was good, the child really enjoyed the game; improved his cognitive skills

Problems: was difficult for him to follow instructions

Case 8: Cerebral palsy

Game: Paint; Reach; concentration colour

Results: Child enjoyed the game very much; helped him with balance; improved walking steps and speed.

Problems: Need support to walk.

Figure 5: Example of observation form by physiotherapist at Inuka CBR from different cases with child patients using the modular tiles system.

\subsection{Regional Hospital: Iringa Hospital}

Interviews and questionnaire was made with the physiotherapist from Iringa Hospital, Walter. At Iringa Hospital, some patients had been somewhat skeptical of using the tiles, since their lack of technological knowledge made them afraid of radiation from the tiles. It took Walter some time to convince the patients, but then most of them did end up trying the tiles, and also finding it very fun, but still having some scare of the technology. The patients made progress, training with the tiles once a week for a couple of weeks.

\subsection{National Hospital: Muhimbili National Hospital}

Interviews were conducted with Director of the Physiotherapy department Denis Rwelamira at Muhimbili National Hospital. Mr. Rwelamira has been using the tiles with a few patients. He uses the games "Side step forward", "walker" and "paint" quite a lot, since these games facilitate that he can teach the patients to walk well, putting the right weight on each step. As cases, we will report the testing with an elderly woman and with two of the children at Muhimbili National Hospital.

\subsubsection{Athetoid Cerebral Palsy}

Michael is 7 years old and suffers from Athetoid Cerebral Palsy, which a type of Cerebral Palsy, which affects the individuals capability to control the muscles (level II). He therefore needs to learn movement control, focus and coordination. While testing the tiles it was evident that he especially had problems with focus and concentration. The tiles and the lights did not catch his attention, even when trying to point at the changing colors. It was therefore also hard for him to make his steps trigger the pressure sensor in the tiles, since he often stepped between the tiles instead of the middle of the tiles. He did have the strength to register his steps, when he placed his foot in correct place on the tiles. 

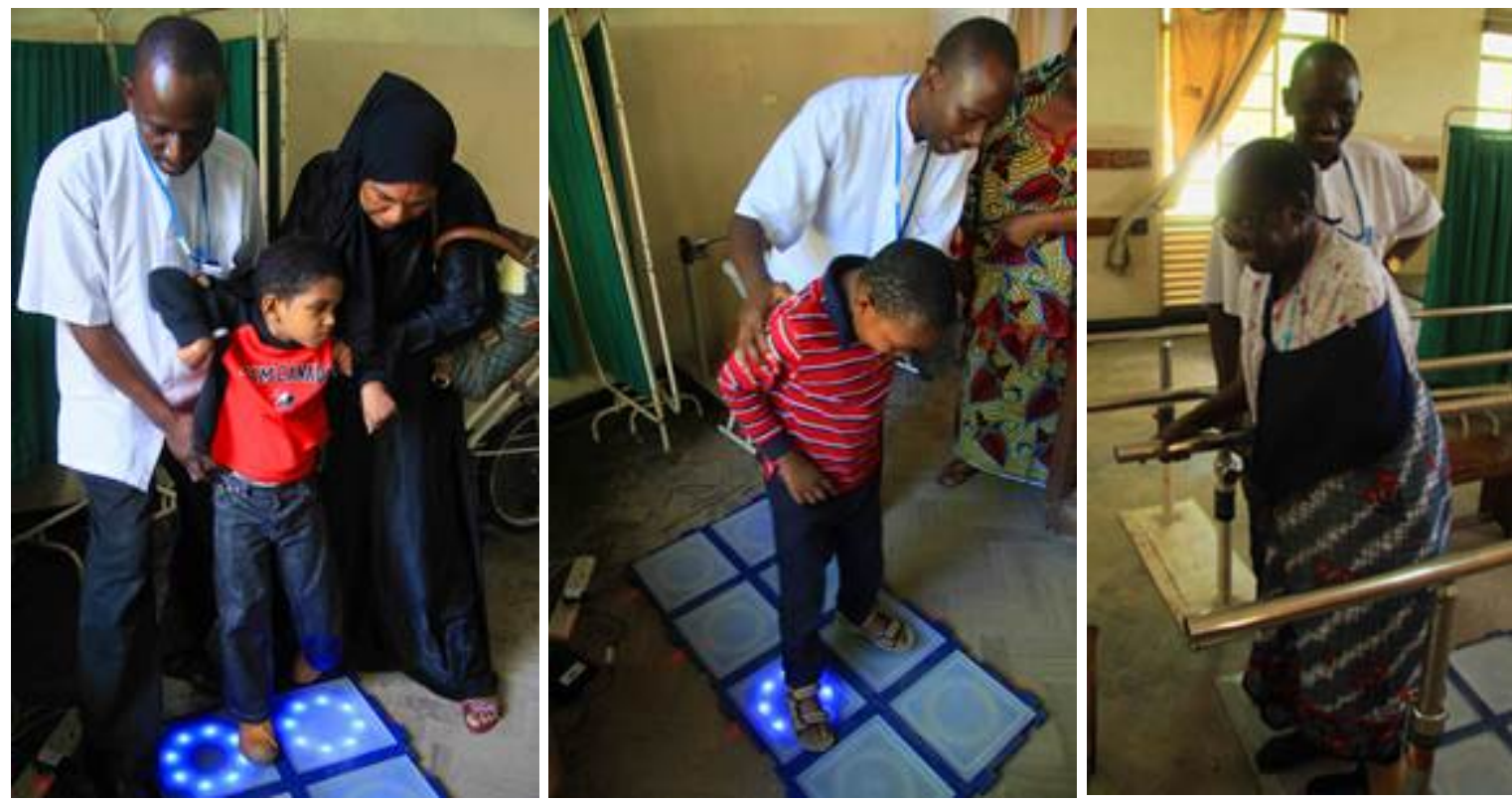

Figure 6: Training on tiles at Muhimbili National Hospital. Left: Boy with Athetoid Cerebral Palsy, Middle: Boy (10y) with Spastic Cerebral Palsy, Right: Lady (72y) with stroke.

\subsubsection{Mild Cerebral Palsy with Slight Mental Disorder}

Ali is 10 years old and suffers from a mild form of Spastic Cerebral Palsy, he needs to work on his balance, but mostly he has to work on concentration, memory and coordination. Since he only has a mild form of CP (level I) he quite quickly gets the idea of the tiles, and how to use them. He is first a bit hesitant, but after a few tries it is clear that he likes it. He gets a few problems; if he does not step correctly on the tile first time, since he then has to concentrate on moving the weight back on that foot. Ali Sadri likes the lights, and understands well how he has to turn off the lights while playing.

\subsubsection{Recovering Stroke Patient}

Paulina is 72 years old and is recovering from a stroke. In everyday life she has to use aids in order to work, and has problems with her left arm. She is exercised in order to improve her concentration, coordination, strength and balance. It is the second time that Paulina uses the tiles, she uses the tiles in combination with a parallel walking bar, because she does not have the strength or the balance to use the tiles on her own. Paulina likes the tiles, because it makes it easier to see whether she is applying weight on the correct foot. Previously, the doctor has told her that she is not putting weight on the right foot, and then Paulina has argued that she was. Now there is no discussion, because the tiles clearly show whether there is weight on the foot or not. This is really encouraging for Paulina. Some of the games such as Side Step Forward, are, however, too fast for Paulina because she cannot process and execute the movement before the light of the tiles has gone out again.

Testing with these users, together with talking to both Mr. Rwelamira and the Senior Occupational Therapist Rosemary Kauzani, gave further insight to the use of the tiles at $\mathrm{MNH}$. A few quotes from the interviews include:

"This can be very good. It works with many different areas, sensory motor, cognition, concentration, and even social skills, when the child is happy about playing”

- Rosemary Kauzani, Senior Occupational Therapist, MNH

"The staff have been of help at my working facility in facilitating direct feedback to my patients exposed to them. The type of patients tested with are 1-Post stroke patients - Who 
need to bear weight on the affected side 2-Children with attention deficit disorder. With children I would appreciate if some modification can be made to induce attractions like sounds and happy activity like clapping and song at the end of the game."

- Denis Rwelamira, Director of physiotherapy department, MNH.

To facilitate such opportunities as pointed out by the director of physiotherapy at the national hospital, we developed and introduced a tablet with an app together with the modular interactive tiles, to allow for game selection, showing of scores, documentation, and sound feedback. At the regional hospital and rehabilitation centres, there were some concern about the fragile screen of the tablet, which should be protected and the cost of the tablet. Further it may be too complicated for the community rehabilitation workers (CRW's), who do not have a lot of education. A tablet seems very technical, so it could potentially be hard to grasp for many of the lower educated workers. With a simple interface and no extra things to take care of, Raya at Inuka CBR evaluates that the tablet would be okay for the rural areas. Otherwise she suggests incorporating some introduction to tablets in the education the CRW's get from Inuka. But in general, as in the national hospital, the health workers saw possibilities in the feedback which the app can provide, also if it was possible to save a patient's progress.

\section{Discussion and Conclusion}

The qualitative studies in the strategic network of national hospital, regional hospitals and rehab centres formed the basis for contextualising the eHealth solution to the health sector in Tanzania. For instance, the studies led to development effort in creating a very simple interface on a tablet app in order to provide the desired score and sound feedback.

Further, the qualitative studies for the contextualisation highlighted that sensitivity of the tiles is especially important for training with children with severe cerebral palsy and other challenges, who may have difficulties in activating the tiles with the force of their hands. Hence, this has led to the investigation of a new mechanical solution for the tiles design. This includes a change of the force sensitive resistor to a mechanical solution around switches to allow a small force (child's hand) to active the tiles.

Also, the qualitative studies, for instance in the Iringa regional hospital, highlighted the challenges of not having a stable electrical energy source. This limits the use of equipment such as treadmills in the rehab gyms, since treadmills need a continuous energy source to drive the motors of the treadmill, so unstable energy provision makes it difficult to plan to use such equipment. Therefore, the tiles are designed to just need very short period of recharging (e.g. 2-3 hours) to provide a long time use (20-30 hours). Indeed, the energy demand of the tiles is less than $1 \%$ of the industry standard of energy use of treadmills, allowing use in rural and deep areas in Sub-Saharan Africa.

As a mHealth solution, the app development will also facilitate collection of data from the rehabilitative play. A main challenge is to provide a user interface which allow any community rehabilitation worker to understand and feel comfortable in using the app and the equipment, hence all data collection will happen in the background. This will facilitate documentation, and lead to quantitative effect studies as a next step: in national hospitals, but potentially also allowing documentation of effect in regional hospitals and rehab centres in rural areas.

As a consequence, this development has resulted in the modular interactive tiles being implemented and used in the daily rehabilitation practice for the last 1.5 years in Inuka rehabilitation centre and community-based rehab, and in Muhimbili National Hospital in Tanzania. 


\section{References}

[1] Aranda-Jan, C. B., Mohutsiwa-Dibe, N., \& Loukanova, S. (2014). Systematic review on what works, what does not work and why of implementation of mobile health (mHealth) projects in Africa. BMC public health, 14(1), 188.

[2] H. H. Lund. "A Concept for a Flexible Rehabilitation Tool for sub-Saharan Africa”. In IST-Africa 2012 Conference Proceedings, Paul Cunningham and Miriam Cunningham (Eds), IIMC International Information Management Corporation, 2012, ISBN: 978-1-905824-34-2

[3] H. H. Lund, L.S.D. Jensen, Y. Ssessanga, R. Abdalahman. ”Implementing Modular Interactive Tiles for Rehabilitation in Tanzania - a Pilot Study", in IST-Africa 2014 Conference Proceedings, Paul Cunningham and Miriam Cunningham (Eds), IIMC International Information Management Corporation, 2014, ISBN: 978-1-905824-43-4

[4] Lieberman, D. A. (1997). Interactive video games for health promotion: Effects on knowledge, selfefficacy, social support, and health.

[5] Griffiths, M. (2005). Video games and health: video gaming is safe for most players and can be useful in health care. BMJ: British Medical Journal, 331(7509), 122.

[6] Göbel, S., Hardy, S., Wendel, V., Mehm, F., \& Steinmetz, R. (2010, October). Serious games for health: personalized exergames. In Proceedings of the international conference on Multimedia (pp. 1663-1666). ACM.

[7] Tanaka K, Parker JR, Baradoy G, Sheehan D, Holash JR, Katz L. A comparison of exergaming interfaces for use in rehabilitation programs and research. J Can Game Stud Assoc. 2012;6(9):69-81

[8] H. H. Lund and R. Beck. "Modular Robotic Tiles: Experiments for Children with Autism”, Artificial Life and Robotics Journal, 13:2, 394-400, 2009.

[9] H. H. Lund "Modular Playware as a Playful Diagnosis Tool for Autistic Children", in Proceedings of IEEE International Conference on Rehabilitation Robotics, IEEE Press, 899-904, 2009.

[10]H. H. Lund, A. Henningsen, and R. Nielsen. ”Modular Robotic System as Multisensory Room in Children's Hospital” In Sugisaka and Takaga (eds.) Proceedings of 14th International Symposium on Artificial Life and Robotics (AROB'14), ISAROB, Oita, 2009.

[11] H. H. Lund "Modular Robotics for Playful Physiotherapy", in Proceedings of IEEE International Conference on Rehabilitation Robotics, IEEE Press, 571-575, 2009.

[12] H. H. Lund. "Modular Interactive Tiles for Rehabilitation - Evidence and Effect”. In Proceedings of 10th Intl. Conf. on Applied Computer Science, WSEAS, Japan, 2010.

[13]H. H. Lund and C. B. Nielsen. "Modularity for Modulating Exercises and Levels - Observations from Cardiac, Stroke, and COLD Patients Therapy”. In Proceedings of 8th International Conference on Ubiquitous Robots and Ambient Intelligence (URAI), IEEE, 2011.

[14]H. H. Lund, and P. Marti. "Designing Modular Robotic Playware". In 18th IEEE International Symposium on Robot and Human Interactive Communication (Ro-Man 2009), IEEE Press, 115-121, 2009.

[15] Dynastreams Innovations Inc. ANT Message Protocol and Usage Document, 2014. http://www.thisisant.com/resources/ant-message-protocol-and-usage (checked: 25/2/2015) 